\title{
Genomic, Biochemical, and Phylogenetic Evaluation of Bacteria Isolated From Deep-sea Sediment Harboring Methane Hydrates
}

Audrey Menegaz Proenca ( $\sim$ proenca.ay@gmail.com)

Pontifical Catholic University of Rio Grande do Sul: Pontificia Universidade Catolica do Rio Grande do Sul https://orcid.org/0000-0002-3747-9719

Maiara Monteiro Oliveira

Pontifical Catholic University of Rio Grande do Sul: Pontificia Universidade Catolica do Rio Grande do Sul

\section{Paula Fernanda Ribas Neves}

Pontifical Catholic University of Rio Grande do Sul: Pontificia Universidade Catolica do Rio Grande do Sul

\section{Adriana Giongo}

Pontifical Catholic University of Rio Grande do Sul: Pontificia Universidade Catolica do Rio Grande do Sul

Rafael Rodrigues de Oliveira

Pontifical Catholic University of Rio Grande do Sul: Pontificia Universidade Catolica do Rio Grande do Sul

\section{Letícia Marconatto}

Pontifical Catholic University of Rio Grande do Sul: Pontificia Universidade Catolica do Rio Grande do Sul

\section{Halesio Milton Correa de Barros Neto}

Petróleo Brasileiro SA: Petrobras

\section{João Marcelo Medina Ketzer}

Linnaeus University: Linneuniversitet

\section{Renata Medina-Silva}

Pontifical Catholic University of Rio Grande do Sul: Pontificia Universidade Catolica do Rio Grande do Sul

\section{Research Article}

Keywords: Cold seeps, deep-sea, marine sediment, microbial ecology, microbiology

Posted Date: November 18th, 2021 
DOI: https://doi.org/10.21203/rs.3.rs-1075050/v1

License: (c) (1) This work is licensed under a Creative Commons Attribution 4.0 International License. Read Full License

Version of Record: A version of this preprint was published at Archives of Microbiology on March 9th, 2022. See the published version at https://doi.org/10.1007/s00203-022-02814-z. 


\section{Abstract}

Over half of the organic carbon on Earth's surface is trapped in marine sediment as methane hydrates. Ocean warming causes hydrate dissociation and methane leakage to the water column, rendering the characterization of microbes from hydrate depositions a pressing matter. Through genomic, phylogenetic, and biochemical assays, we characterize the first microorganisms isolated from the Rio Grande Cone (Brazil), reservoir responsible for massive methane releases to the water column. From sediment harboring rich benthic communities, we obtained 43 strains of Brevibacillus sp., Paenibacillus sp. and groups of Bacillus sp. Methane-enriched samples yielded strains of the Pseudomonas fluorescens complex, exhibiting fluorescent siderophore production and broad multi-carbon catabolism. Genomic characterization of a novel Pseudomonas sp. strain indicated 32 genes not identified in the closest related type-species, including mercury resistance proteins. Our results provide phylogenetic and genomic insights on the first bacterial isolates retrieved from a poorly explored region of the South Atlantic Ocean.

\section{Introduction}

Marine sediments of continental margins around the globe store considerable mass of methane ( 0.5 to $12.7 \times 10^{21} \mathrm{~g}$ ) in the form of gas hydrates (Dickens 2011; Piñero et al. 2013; Ketzer et al. 2019). Hydrates are crystalline structures formed by gas and water molecules, which remain stable in zones of low temperature and high pressure within deep-sea sediment. Increases in temperature or decreases in pressure (sea-level fall) lead to hydrate dissociation and consequential release of methane to the ocean (Giustiniani et al. 2013; Hunter et al. 2013). As these environmental changes could greatly impact the microbial basis of deep-sea communities, characterizing microbes directly associated with methane reservoirs is a pressing matter.

While methane-oxidizing microbes, as primary producers of these communities, are at the center of most characterization efforts targeting cold seep environments, the metabolic potential of heterotrophic bacteria from these sites is often overlooked. These heterotrophs represent an essential link between primary production and higher trophic levels, growing seafloor biofilms that feed benthic fauna and ciliates (Takishita et al. 2010; Niemann et al. 2013). Moreover, previous studies have shown that heterotrophic bacteria can enhance growth and activity of methanotrophs in mixed cultures (Iguchi et al. 2011; Stock et al. 2013; Ho et al. 2014; Veraart et al. 2018), while metabolizing toxic by-products of methane oxidation and generating industrially relevant bioproducts (Singh et al. 2019). As such, the isolation and characterization of heterotrophs from methane-rich sediments could contribute for the understanding of associated microbial communities and future biotechnological applications.

A recently characterized gas hydrate province in the South Atlantic Ocean, the Rio Grande Cone (Miller et al. 2015; Ketzer et al. 2019), offers a virtually unexplored microbiological potential. This geological feature of the Brazilian continental slope possibly harbors 22 trillion $\mathrm{m}^{3}$ of methane in hydrates (Ketzer et al. 2019), with methane high flux and seepage below seafloor reported from pore-water analyses (Rodrigues et al. 2017). Chemical and isotopic signatures revealed a biogenic origin for the methane in 
hydrates from this region (Miller et al. 2015), indicating the activity of methanogenic archaea in the sediment (Giongo et al. 2016). However, massive methane releases due to contemporary ocean warming are already underway in this reservoir (Ketzer et al. 2020), in a volume that is estimated to surpass the capacity of microbial oxidation within the sediment. The characterization of microbes present in these sediments has so far been restricted to $16 \mathrm{~S}$ amplicon sequencing, which has shown that a diverse array of microorganisms fuel complex benthic communities in the Rio Grande Cone (Giongo et al. 2016; Medina-Silva et al. 2018b). However, the metabolic and genomic potential of microbial isolates from this reservoir remains unknown.

Through biochemical, phylogenetic, and genomic techniques, this study presents a comprehensive characterization of the first bacterial isolates obtained from hydrate-rich sediments of the Rio Grande Cone. Cultivation efforts resulted in the isolation of diverse Bacilli species from a pockmark with associated benthic fauna, and Pseudomonas sp. isolates were obtained from enrichment cultures with methane as sole carbon source. These siderophore-producing bacteria were identified as members of the Pseudomonas fluorescens complex, showing broad metabolic capacity. Whole genome characterization of a novel Pseudomonas sp. strain revealed 32 protein coding sequences absent in its closest 16S rRNA gene relatives, including mercury resistance genes. Our results contribute to the genetic and metabolic characterization of heterotrophic microbes thriving in a newly described methane hydrate reservoir on the South Atlantic Ocean.

\section{Materials And Methods}

\section{The Rio Grande Cone}

Samples were obtained in the Rio Grande Cone (RGC) gas hydrate province, Pelotas Basin, western South Atlantic Ocean (Fig. 1). The RGC consists of a structure within the basin that contains two extensive pockmark fields, in which gas hydrates have been recovered (Miller et al. 2015; Ketzer et al. 2019). Six cores were selected for microbiological analysis (Fig. 1, Table 1) based on the presence of methane hydrates or association with previously described chemosynthetic communities (Giongo et al. 2016). Background sites were selected outside of pockmarks and had no hydrates. Methane and ammonia concentrations were determined as previously described (Giongo et al. 2016).

\section{Sediment sampling and storage}

Sediment samples were collected through piston coring and dredging (van Veen grab sampler). Collections were performed during two oceanographic expeditions. Two piston cores (PC44 and PC49) and a dredge sample (DR44) were collected in the first expedition (MR11; February 2011) on the Brazilian research vessel Marechal Rondon, equipped with a $6 \mathrm{~m}$ long piston corer. Four other cores (PC80, PC81, PC84, PC109) were retrieved during the second expedition (MD195; July 2013) onboard the French research vessel Marion Dufresne, equipped with a 20-40 m long Calypso piston corer. Sediment cores consisted of dark green to dark brown mud with hydrate laminae and carbonate concretions (Miller et al. 2015). $20 \mathrm{~g}$ of sediment were collected for each sample (detailed in Table 1) for microbiological analysis. 
Samples targeting the culture of fast-growing bacteria were placed in $15 \mathrm{ml}$ falcon tubes containing $30 \%$ glycerol and $70 \%$ seawater, and stored at $-80^{\circ} \mathrm{C}$. Samples targeting bacteria with methanotrophic potential were stored in $20 \mathrm{ml}$ vials filled with argon gas and stored at $4^{\circ} \mathrm{C}$.

\section{Microbial culturing and isolation}

Bacterial isolates were obtained through a combination of sediment culturing approaches. Three distinct culture media were used for enrichment and culturing: BHI (brain-heart infusion) and YPD (yeast extractpeptone-dextrose), both rich media for heterotrophs, and NMS (nitrate mineral salts), which provides no carbon source. Replicates were incubated at room temperature, $30^{\circ} \mathrm{C}$, or $37^{\circ} \mathrm{C}$. Incubation at $4^{\circ} \mathrm{C}$, matching the sediment surface temperature, was tested for 8 weeks without success, although it allowed for continued growth of cultures that started at higher temperatures.

A portion of sediment from MR11 samples were enriched prior to isolation by inoculating $200 \mu$ sediment into BHI, YPD, or NMS broth, incubated for $48 \mathrm{~h}$ under $140 \mathrm{rpm}$ agitation. Bacteria present in enriched samples were isolated in BHI, YPD, or NMS-agar plates, with incubation time ranging from $24 \mathrm{~h}$ to 4 weeks depending on the temperature and nutritional source. For direct sediment plating, another aliquot of sediment was retrieved from the original sample and incubated on BHI, YPD, or NMS-agar plates for 7 days. Direct sediment culture plates were placed in airtight containers injected with methane gas and incubated at room temperature until colonies were observed. Isolates obtained through this method were stored at $-80^{\circ} \mathrm{C}$ with $30 \%$ glycerol.

MR11-PC49 and MD195 samples, which were mostly obtained from sediments containing methane hydrates and plumes (Table 1), were subjected to enrichment culture focusing on methane-related metabolisms. Sediment samples were inoculated into $20 \mathrm{ml}$ vials containing $5 \mathrm{ml} \mathrm{NMS}$-broth, and each vial was injected with methane gas provided as sole carbon source. After three weeks of enrichment, samples were diluted to extinction and isolates were cultured in NMS-broth with methane for another three weeks. Isolation was verified by plating on NMS-agar, incubated in aerobic airtight containers with methane gas until colonies appeared. Isolates obtained through this method were stored at $-80^{\circ} \mathrm{C}$ with $5 \%$ Dimethyl Sulfoxide (DMSO). All isolates were characterized by Gram staining under optical microscopy $(1000 x)$, to confirm their morphology and culture purity.

\section{Biochemical profiling}

Bacterial isolates obtained through dilution to extinction and methane exposure were evaluated according to presence or absence of growth on multicarbon substrates. For this assay, performed in triplicates, NMS-broth was supplemented with $1 \%$ of either compound: glucose, fructose, sucrose, maltose, arabinose, mannitol, ethanol, sodium acetate or ammonium acetate. Isolates were inoculated in the supplemented broth and cultured for up to 5 days at $28^{\circ} \mathrm{C}$, without methane.

\section{DNA extraction and sequencing}


All isolates were subjected to molecular taxonomic identification. DNA extraction was performed as previously described (Pitcher et al. 1989). Briefly, $500 \mu \mathrm{L}$ of each culture were centrifuged at $8150 \mathrm{~g}$ for 10 min and resuspended in $1 \mathrm{M} \mathrm{NaCl}$. After nucleic acid solubilization in TE (10 mM Tris + $1 \mathrm{mM}$ EDTA), 500 $\mu \mathrm{L}$ guanidinium thiocyanate and $250 \mu \mathrm{L} 7.5 \mathrm{M}$ ammonium acetate were employed in the cell lysis and protein denaturation. To segregate nucleic acids and denatured proteins in different solution phases, 24:1 chloroform: isoamyl alcohol was added, and the supernatant phase was transferred to a centrifuge tube containing isopropyl alcohol. Isopropyl-precipitated DNA was then washed with $70 \%$ ethanol for removing salts and dissolved in TE for storage at $-80^{\circ} \mathrm{C}$.

Molecular characterization was performed through PCR amplification and Sanger sequencing of $16 \mathrm{~S}$ rRNA genes. DNA extracted from MR11 isolates was amplified using primers 515F and 806R (Bates et al. 2011), which target the V4 domain. DNA from MD195 and four MR11 isolates (MR06, MR23, MR34, and MR43) was amplified using primers $9 \mathrm{~F}$ and 1542R (Edwards et al. 1989), targeting mostly the full-length 16S rRNA gene. PCR reaction mixture $(20 \mu \mathrm{L})$ contained $2 \mu \mathrm{L}$ of each primer, $2 \mu \mathrm{L} \mathrm{MgCl} 2$ buffer, $2 \mu \mathrm{L}$ dNTP, $0.8 \mu \mathrm{L} \mathrm{MgCl}_{2}, 0.2 \mu \mathrm{L}$ Taq DNA Polymerase (Invitrogen), and $1 \mu \mathrm{L}$ DNA. PCR conditions consisted of an initial denaturation at $94^{\circ} \mathrm{C}$ for $2 \mathrm{~min}$, followed by 25 cycles of $94^{\circ} \mathrm{C}$ for $45 \mathrm{~s}, 55^{\circ} \mathrm{C}$ for $45 \mathrm{~s}$, and $72^{\circ} \mathrm{C}$ for $60 \mathrm{~s}$, with a final extension of $72^{\circ} \mathrm{C}$ for $6 \mathrm{~min}$. Amplified DNA samples were sequenced using a MegaBACE1000 automated sequencer (GE Healthcare) by the Genomic and Molecular Biology Laboratory (PUCRS, Brazil).

\section{Molecular identification and phylogenetic analysis}

16S rRNA gene sequences were trimmed using Unipro UGENE v. 33 (Okonechnikov et al. 2012) and compared to the EzBioCloud curated database (Yoon et al. 2017) for the identification of bacterial isolates. Reference sequences were downloaded into EzEditor (Jeon et al. 2014) for alignment visualization. Best matching reference sequences and trimmed isolate sequences were used for phylogenetic tree construction on MEGA X (Kumar et al. 2018). Multiple sequence alignment was performed using MUSCLE, and trees were built using the Maximum Likelihood method with 1,000x bootstrap.

\section{Draft genome sequencing and assembly}

Isolate MD195-PC81-125, obtained through dilution-to-extinction cultures with methane as sole carbon source, was selected for whole genome sequencing after initial 16S rRNA phylogenetic analyses. Genomic DNA extraction was performed on an overnight culture using MagMAX Nucleic Acid Isolation Kit (Thermo Fisher Scientific), according to manufacturer's instructions, obtaining $6 \mu \mathrm{g}$ of DNA (Qubit Fluorometric Quantification, Thermo Fisher Scientific). A $100 \mu \mathrm{l} \mathrm{sample} \mathrm{of} 10 \mathrm{ng} / \mathrm{ml}$ DNA was sonicated with four cycles, and 250 bp fragments were selected (in 1\% agarose gel electrophoresis) for sequencing. Sequencing was performed using an Ion PGM System, yielding 1,644,499 single-end reads. Read quality was assessed using FastQC on Unipro UGENE v. 33. De novo genome assembly was performed with MIRA v. 4.0 (Chevreux et al. 1999) through lonGAP (Baez-Ortega et al. 2015), with lon 
Torrent parameters specified for assembly (minimum read length $=40$; minimum reads per contig $=5$ ), clipping (quality control minimum quality $=20$; quality control window length $=30$ ) and alignment ( minimum overlap $=19$; minimum score $=15$; minimum relative score $=70)$. A total of $6,481,483$ bp were assembled into 1,056 contigs ( $>500$ bp each; $N 50=13,470$ ) with $27 x$ sequencing depth coverage, which were subsequently aligned and reordered on Mauve (Darling et al. 2004) against reference sequences previously selected via $16 \mathrm{~S}$ rRNA similarity. To further ensure the purity of this assembly, contigs were entered into the ContEst16S tool available on EzBioCloud (Yoon et al. 2017), which reported no contaminants. Genome completeness analysis was performed using BUSCO v. 5.0 on Galaxy v. 21.05.rc1 (Goecks et al. 2010).

\section{Genome annotation}

Protein coding sequence identification and functional annotations were performed using the NCBI Prokaryotic Genome Annotation Pipeline (NCBI PGAP) (Tatusova et al. 2016) and RAST v.2.0 (Aziz et al. 2008). Because posterior analysis revealed misannotations in the RAST output, we decided to use NCBI PGAP annotations alone, despite yielding fewer protein coding sequences. Regions coding for proteins of particular interest were further analysed using NCBI CD-Search (Marchler-Bauer and Bryant 2004) and BLAST searches against reviewed entries of the UniProtKB/SwissProt database and RefSeq protein database.

Coding sequences identified through the NCBI PGAP were submitted to EggNOG Mapper v.2 (eggNOG database v.5.0) (Huerta-Cepas et al. 2016), for the determination of Cluster of Orthologous Genes (COG) classes and KEGG Orthologs (Kanehisa et al. 2016). The resulting functional annotation was compared with the COG distribution and KEGG classification of type strains retrieved from EzBioCloud (Yoon et al. 2017). Although EzBioCloud provides complete COG annotations for all genomes, we have opted for de novo mapping of all references using EggNOG Mapper to maintain methodological consistency. Statistical analysis was performed using R v.3.6.1 (R Core Team 2017). The assembled genome is available from NCBI BioProject PRJNA616366.

\section{Results}

\section{Molecular identification and phylogenetic analysis of isolates from sediment with associated benthic fauna}

To characterize diverse microbial isolates from deep-sea sediment, we began by exploring a location (PC44/DR44) with associated tubeworms and bivalve mollusks, previously identified as markers of chemosynthetic microbial activity at the site (Giongo et al. 2016). As the presence of benthic fauna suggests a connection with higher trophic levels and a larger concentration of total organic matter, this site was particularly interesting for the isolation of heterotrophs. The sediment surface at PC44 exhibited low methane concentrations (Table 1), although another site within close proximity (PC49) contained hydrates. Samples were subjected to a combination of culturing approaches focusing on the obtention of fast-growing aerobic strains (Supplementary Table 1). 
The resulting 40 isolates were identified through sequencing of the 16S rRNA gene (Supplementary Table 1). The genus Bacillus comprised most isolates $(n=34)$, likely favored by enrichment in nutrient-rich media. Our phylogenetic analysis categorized these bacteria into four groups (Fig. 2), three of which comprised species of high 16S rRNA similarity $(>99 \%)$ : the $B$. pumilus group $(\mathrm{n}=19)$, the $B$. megaterium group $(n=7)$, and the $B$. cereus group $(n=4)$. The $B$. pumilus group included most of our isolates, along with marine and soil reference strains often indistinguishable at the $16 \mathrm{~S}$ sequence level. The $B$. cereus group included four isolates with V4 subregion identical (MR25, MR38, MR39) or highly similar (MR26, 99.5\%) to reference species in this group, such as $B$. mycoides and $B$. thuringienses. The fourth Bacillus group comprised three isolates (MR46, MR49, and MR50) equally similar to $B$. simplex, $B$. halmapalus and Brevibacterium frigoritolerans, and a fourth isolate (MR40) displaying $100 \%$ similarity to Bacillus gossypii.

The genus Paenibacillus comprised five of the remaining isolates. Interestingly, all were obtained through direct plating of sediment samples, with four of them being found in plates exposed to methane gas (Supplementary Table 1). Strains MR07, MR08, and MR10 showed highest similarity to $P$. typhae, while MR01 and MR14 were 100\% similar to both P. panacisoli and P. massiliensis. MR01 cells exhibited filamentous morphology when growing on NMS agar at room temperature. Filamentous cells have been observed in P. vortex (Vallotton 2013), driving colony expansion and creating complex colony patterns. Although vortex-like patterns were not observed for MR01 growing in NMS agar, the phenotype could be culture media-dependent. Finally, one isolate (MR16) had 100\% similarity with Brevibacillus laterosporus. Taken together, our results indicate the presence of diverse heterotrophic Bacillales in sediment with a thriving benthic fauna.

\section{Broad metabolic potential of bacteria isolated with methane gas}

To target the isolation of organisms associated with methane hydrates, we performed enrichment of sediment samples with methane gas as the sole carbon source. These samples originated from five collection sites where methane hydrates or plumes were observed (Table 1). We enriched sediment in vials containing NMS-broth, injected with methane gas and cultured at room temperature for up to three weeks. Enriched broth was diluted to extinction and plated on NMS-agar incubated with methane, yielding 14 bacterial isolates.

To investigate the metabolic potential of these isolates, we evaluated their ability to grow on compounds containing C-C bonds. We performed a growth assay by supplementing NMS-broth with $1 \%$ of a multicarbon substrate, either a saccharide, alcohol or acetate (Fig. 3). The results revealed a broad catabolic potential among the isolates, with most sugars yielding growth for all samples. Five isolates did not grow in arabinose, and four tests showed no growth in ammonium acetate. Eight isolates produced a yellowish-green fluorescent pigment when grown in various multicarbon sources (highlighted on Fig. 3). These results suggest that, despite being isolated from methane enrichment of sediment samples, these organisms consisted of generalist bacteria (i.e. adapted to a variety of carbon sources), some of which are able to synthesize fluorescent molecules. 


\section{Taxonomic and phylogenetic classification of methane-grown isolates}

To further characterize organisms obtained from methane enrichment, we performed the taxonomic identification of our isolates (Supplementary Table 2). Strains isolated from PC49, for which we obtained partial 16S rRNA sequences, were included in the MR11 phylogenetic tree in Fig. 2. Strains MET16 and MET17 showed highest similarity to Paenibacillus oceanisediminis, forming a close branch $(>99 \%$ similarity) with the methanotrophic species Paenibacillus illinoisensis.

We identified the eight fluorescence-producing isolates as Pseudomonas sp. (Supplementary Table 2, Fig. 4). Seven of these isolates were grouped together, showing high similarity to $P$. rhodesiae, $P$. poae, $P$. trivialis and other closely related species. These bacteria are part of the $P$. fluorescens complex (GarridoSanz et al. 2017), comprising species capable of producing fluorescent siderophores such as pyoverdine. Isolate MD195-PC81-125, however, was separately grouped from the other strains, showing higher similarity to $P$. koreensis and Pseudomonas sp. PDKZ.

\section{Genomic characterization of Pseudomonas MD195-PC81-125}

The distinct grouping of strain MD195-PC81-125 motivated further characterization of this isolate through whole genome sequencing. The genome assembly resulted in a $6.4 \mathrm{Mbp}$ draft genome comprising 1,056 contigs, with a G+C content of $59.7 \%$ and estimated completeness of $88.6 \%$. A total of 4,686 protein coding sequences were identified and annotated through the NCBI Prokaryotic Genome Annotation Pipeline. Among these genes, $92.15 \%$ were functionally characterized according to Clusters of Orthologous Groups (COG), and 64.32\% were successfully assigned a KEGG Orthology (KO) class.

To visualize and quantify the relative abundance of COG classes found in MD195-PC81-125, we compared it to the genomes of closely related Pseudomonas spp. (Fig. 5). For each type species, we considered the proportion of genes assigned to each COG category. The overall pattern of COG proportions exhibited by MD195-PC81-125 shared a similar profile with the closest Pseudomonas spp. (NRST, PDKZ and $P$. koreensis), indicated by the phylogenetic analysis on Fig. 5. Compared to all type strains, MD195-PC81-125 encoded a significantly lower gene proportion in three COG classes: (L) replication and repair (3.27\%, binomial test, $p=0.015),(\mathrm{G})$ carbohydrate transport and metabolism $(4.10 \%, p=0.036)$, and (I) lipid transport and metabolism $(3.37 \%, p=0.043)$. On the other hand, this marine strain exhibited a greater proportion of genes with unknown function $(S ; 22.17 \%, p<0.001)$. These coding sequences represent potential targets for future metabolic explorations.

To further investigate the differences between protein coding sequences of MD195-PC81-125 and its close relatives, we considered the KEGG Orthologs present in the reference genomes of type strains $P$. koreensis LGM21318, PDKZ FDAARGOS 376 and NRST TYU6. A total of 32 KEGG Orthologs observed in MD195-PC81-125 were not present in the genomes of these similar strains (Supplementary Table 3). Among these, we identified genes from the mercury resistance operon (merA, merE, merPand $m e r T$ ) and a nickel/cobalt efflux protein (RcnA). The COG categories assigned to these 32 unique KEGG classes 
indicated 4 coding sequences associated with replication and repair, 4 involved in inorganic ion transport and metabolism, and 12 of unknown function.

Regarding genes that could favor its growth in methane-rich environments, we investigated isolate MD195-PC81-125 for the genetic encoding of methylotrophy enzymes. No evidence of a methane monooxygenase (MMO) enzyme gene was observed, although EggNOG annotations identified a putative ammonia monooxygenase (AMO), which shared $98.2 \%$ identity with AMO of other Pseudomonas spp. (RefSeq protein database). Because these enzymes share a close evolutionary history, AMO is capable of oxidizing methane, albeit with a lower affinity than ammonia (Ross and Rosenzweig 2017). However, a closer inspection of this putative AMO sequence indicated that it shares only $13 \%$ similarity with the AMO encoded by Nitrosomonas europaea (UniProt/Swiss Prot accession Q04507). The enzyme encoded by MD195-PC81-125 exhibited a conserved AbrB family domain, which is also present in AMO database entries of heterotrophic nitrifying bacteria such as Paracoccus denitrificans (A1BBW9, UniProtKB/TrEMBL), Alcaligenes faecalis (CUI72289.1, GenBank), and Proteus mirabilis (S4V944, UniProtKB/TrEMBL). As it is still necessary to determine whether these database entries derived from a past misannotation of AbrB proteins as AMO, we have decided not to annotate our entry as AMO.

Regarding the ability to metabolize other compounds without C-C bonds, strain MD195-PC81-125 might be capable of coupling carbon and sulfur uptake through the oxidation of dimethylsulfone (Supplementary Table 4), an abundant compound in marine environments. This process is mediated by the enzyme dimethyl sulfone monooxygenase (SfnG), which was identified in MD195-PC81-125 as sharing $95.4 \%$ similarity with reference P. fluorescens SfnG (UniProtKB/Swiss-Prot, SFNG_PSEPF). This oxidation results in methanesulfonate, which is oxidized into sulfide and formaldehyde by alkanesulfonate monooxygenase (SsuD; 93.7\% identity with Pseudomonas savastanoi SSUD_PSEPK, UniProtKB/SwissProt). While diverse bacteria employ this pathway for sulfur uptake, MD195-PC81-125 also encodes the enzymes formaldehyde dehydrogenase (FdhA) and formate dehydrogenase (FdoGHI), thus completing the $\mathrm{C} 1$ dissimilatory pathway with the release of carbon dioxide (Supplementary Table 4). Taken together, these results characterize MD195-PC81-125 as a deep-sea Pseudomonas sp. strain with broad metabolic potential.

Table 1. Characterization of sampling sites according to methane-related features and chemical analyses. Background cores were collected outside pockmarks and contained no hydrates. 


\begin{tabular}{|c|c|c|c|c|c|c|}
\hline Expedition & $\begin{array}{l}\text { Piston } \\
\text { core \# }\end{array}$ & $\begin{array}{l}\text { Water } \\
\text { column } \\
\text { (m) }\end{array}$ & Features & $\begin{array}{l}\text { Sampling } \\
\text { depth (mbsf) }\end{array}$ & $\begin{array}{l}\text { Ammonia } \\
\text { (mg/L) }\end{array}$ & $\begin{array}{l}\text { Methane } \\
(\operatorname{ppm}(\mathrm{v} / \mathrm{v}))\end{array}$ \\
\hline MR11 & PC 44 & 1,297 & $\begin{array}{l}\text { Benthic } \\
\text { fauna }\end{array}$ & 0 & - & 250 \\
\hline MR11 & PC 49 & 1,200 & $\begin{array}{l}\text { Methane } \\
\text { hydrates }\end{array}$ & 0 & 0.5 & 1,823 \\
\hline \multirow[t]{2}{*}{ MD195 } & \multirow[t]{2}{*}{ PC 80} & \multirow[t]{2}{*}{1,438} & \multirow[t]{2}{*}{ Background } & 0 & 0.4 & 2,745 \\
\hline & & & & 3 & 5.4 & 2,376 \\
\hline \multirow[t]{2}{*}{ MD195 } & \multirow[t]{2}{*}{ PC 81} & \multirow[t]{2}{*}{1,369} & \multirow[t]{2}{*}{ Background } & 0 & 0.9 & 1,908 \\
\hline & & & & 3 & 2.3 & 1,881 \\
\hline \multirow[t]{2}{*}{ MD195 } & \multirow[t]{2}{*}{ PC 84} & \multirow[t]{2}{*}{1,373} & \multirow{2}{*}{$\begin{array}{l}\text { Methane } \\
\text { hydrates }\end{array}$} & 0 & 6.9 & - \\
\hline & & & & 4.11 & 24.5 & 39,577 \\
\hline \multirow[t]{2}{*}{ MD195 } & \multirow[t]{2}{*}{ PC 109} & \multirow[t]{2}{*}{1,229} & \multirow{2}{*}{$\begin{array}{l}\text { Methane } \\
\text { plume }\end{array}$} & 0 & 0.6 & 7,866 \\
\hline & & & & 3 & 0.6 & 12,776 \\
\hline
\end{tabular}

MR11 = Marechal Rondon, 2011

MD195 = Marion Dufresne, 2013

mbsf $=$ meters below sea floor

\section{Discussion}

The Rio Grande Cone stands as a recently described gas hydrate province on which a massive release of methane from deep-sea sediment has been verified (Ketzer et al. 2020). This gas seepage could be worsened by ongoing warming of ocean waters (Giustiniani et al. 2013; Hunter et al. 2013; Piñero et al. 2013), potentially altering the composition of biological communities in the area. As such, characterizing deep-sea microbial communities in the Rio Grande Cone is essential for determining the baseline environmental functioning in the area before further hydrate dissociation changes these patterns. As part of a multi-disciplinary effort to explore the Rio Grande Cone (Miller et al. 2015; Giongo et al. 2016; Medina-Silva et al. 2018b), this study presented a phylogenetic, genomic, and physiological characterization of heterotrophic microbes isolated from a methane-rich marine reservoir.

A broad metabolic potential was found in the diverse isolates obtained from the Rio Grande Cone. The present study has focused on obtaining fast-growing aerobic isolates, mostly from the sediment surface. From rich-media cultures we obtained 40 isolates of Bacillales, including the genus Bacillus, Brevibacillus and Paenibacillus, all with known marine diversity (Ettoumi et al. 2013; Lee et al. 2013; Jhala et al. 2014; Zheng et al. 2014). These isolates were obtained from site PC44, located within a 
pockmark with thriving benthic fauna, including Acharax sp. bivalve mollusks (Giongo et al. 2016) and Escarpia sp. tubeworms (Medina-Silva et al. 2018b). Although no 16S rRNA amplicon sequencing data is available for PC44, our previous study of the nearby site PC48 identified over $40 \%$ of the prokaryotes present in the sediment surface as Firmicutes (Medina-Silva et al. 2018b), thus in agreement with the high yield of Bacillales in the current study. Previous investigations in similar systems have reported a higher relative frequency of Bacillales (and other Firmicutes) in sediments harboring hydrates, when compared to hydrate-less samples from the same area (Cui et al. 2020). Moreover, the high representation of Firmicutes in our samples could be explained their ability to form endospores, which have been shown to create a large pool of culturable microbes in deep-sea sediment (Wörmer et al. 2019).

This study also obtained seven fluorescent siderophore-producing isolates of the $P$. fluorescens complex, one of the most diverse Pseudomonas sp. groups (Garrido-Sanz et al. 2017) and which have a strong bioremediation potential (Wasi et al. 2013). These isolates were cultured through enrichments of deepsea sediment with methane as sole carbon source, targeting aerobic bacteria that could be involved with hydrates and methanotrophs present in the sediment. Besides representing ubiquitous microbes, previous sampling efforts in the same area of the Rio Grande Cone identified Pseudomonas as the $9^{\text {th }}$ most abundant prokaryotic genus in bottom waters, comprising up to $14.9 \%$ of the identified OTUs at one of the sites (Medina-Silva et al. 2018a). Previous studies performing methane enrichment of environmental samples have also obtained heterotrophic Bacillus and Pseudomonas isolates (Veraart et al. 2018), bacteria that can influence growth and activity of methanotrophs (Stock et al. 2013; Ho et al. 2014; Veraart et al. 2018). Furthermore, experiments have shown that biosurfactants produced by Pseudomonas aeruginosa and Bacillus subtilis improve the formation kinetics of gas hydrates (Rogers et al. 2003; Jadav et al. 2017). The isolation of similar organisms directly from hydrate-bearing sediments thus provides a potential biotechnological resource for further exploration of biosurfactants.

Among the isolates obtained in this study, genomic analysis of Pseudomonas MD195-PC81-125 revealed genes related to carbon and sulfur uptake, which seem to occur as an adaptation to methane-rich environments. Moreover, four mercury resistance genes absent in closely related Pseudomonas spp. (Supplementary Table 3) were also detected. These coding sequences belonged to the meroperon, encoding proteins that were recently described in the marine strain Pseudomonas stutzeri 273 as mercury transporters (MerE, MerT, MerP) and mercuric reductase (MerA) (Zheng et al. 2018). Further investigations of Pseudomonas MD195-PC81-125 will determine the level of mercury resistance exhibited by this isolate.

Our study highlights the broad metabolic potential of heterotrophic microbial isolates from the Rio Grande Cone, providing insights on the first bacteria cultured from the reservoir. These observations provide a baseline for future experimental approaches, targeting the elucidation of links between methane oxidation and other members of the microbial communities associated with deep-sea sediments harboring gas hydrates.

\section{Declarations}


Funding: This work was supported by PETROBRAS through the Conegas Project, the Institute of Petroleum and Natural Resources research fund, and the Academic Excellence Program by the Pontifical Catholic University of Rio Grande do Sul. AMP was supported by the Program for Institutional Internationalization (PrInt) Postdoctoral Fellowship provided by the CAPES Foundation, Ministry of Education, Brazil.

Conflicts of interest: Author HMCBN is employed by the company PETROBRAS. The authors declare that the research was conducted in the absence of any commercial or financial relationships that could be construed as a potential conflict of interest.

Availability of data and material: The datasets generated during the current study are available in the GenBank repository, for which accession numbers are provided in Methods, Supplementary Table 1, and Supplementary Table 2.

Code availability: Not applicable.

Author contributions: All authors contributed to the study conceptualization and design. Preparation of materials and experimental procedures were performed by AMP, MMO, PFRN, AG, RRO, and LM. HMCBN, JMMK, and RMS acquired funding and provided resources and supervision. AMP and RMS drafted the manuscript, which was revised and approved by all authors.

\section{Acknowledgments}

We thank colleagues at the Institute of Petroleum and Natural Resources (IPR-PUCRS) and the Immunology and Microbiology Laboratory for support and fruitful discussions. Particularly, we thank the IPR Geochemical Analysis Lab for technical assistance with methane cultures and the numerous contributors who helped preparing fieldwork materials.

\section{References}

Aziz RK, Bartels D, Best A, et al (2008) The RAST Server: Rapid annotations using subsystems technology. BMC Genomics 9:1-15. https://doi.org/10.1186/1471-2164-9-75

Baez-Ortega A, Lorenzo-Diaz F, Hernandez M, et al (2015) lonGAP: Integrative bacterial genome analysis for Ion Torrent sequence data. Bioinformatics 31:2870-2873.

https://doi.org/10.1093/bioinformatics/btv283

Bates ST, Berg-Lyons D, Caporaso JG, et al (2011) Examining the global distribution of dominant archaeal populations in soil. ISME J 5:908-917. https://doi.org/10.1038/ismej.2010.171

Chevreux B, Wetter T, Suhai S (1999) Genome Sequence Assembly Using Trace Signals and Additional Sequence Information. Comput Sci Biol Proc Ger Conf Bioinforma 99:45-56.

https://doi.org/10.1.1.23/7465 
Cui H, Su X, Liang J, et al (2020) Microbial diversity in fracture and pore filling gas hydrate-bearing sediments at Site GMGS2-16 in the Pearl River Mouth Basin, the South China Sea. Mar Geol 427:106264. https://doi.org/10.1016/j.margeo.2020.106264

Darling ACE, Mau B, Blattner FR, Perna NT (2004) Mauve: Multiple alignment of conserved genomic sequence with rearrangements. Genome Res 14:1394-1403. https://doi.org/10.1101/gr.2289704.tion

Dickens GR (2011) Down the Rabbit Hole: Toward appropriate discussion of methane release from gas hydrate systems during the Paleocene-Eocene thermal maximum and other past hyperthermal events. Clim Past 7:831-846. https://doi.org/10.5194/cp-7-831-2011

Edwards U, Rogall T, Blöcker H, et al (1989) Isolation and direct complete nucleotide determination of entire genes. Characterization of a gene coding for 16 S ribosomal RNA. Nucleic Acids Res 17:7843-7853

Ettoumi B, Guesmi A, Brusetti L, et al (2013) Microdiversity of deep-sea Bacillales Isolated from Tyrrhenian Sea sediments as revealed by ARISA, 16S rRNA gene sequencing and BOX-PCR fingerprinting. Microbes Environ 28:361-369. https://doi.org/10.1264/jsme2.ME13013

Garrido-Sanz D, Arrebola E, Martínez-Granero F, et al (2017) Classification of isolates from the Pseudomonas fluorescens complex into phylogenomic groups based in group-specific markers. Front Microbiol 8:1-10. https://doi.org/10.3389/fmicb.2017.00413

Giongo A, Haag T, Simão TLL, et al (2016) Discovery of a chemosynthesis-based community in the western South Atlantic Ocean. Deep Res Part I Oceanogr Res Pap 112:45-56.

https://doi.org/10.1016/j.dsr.2015.10.010

Giustiniani M, Tinivella U, Jakobsson M, Rebesco M (2013) Arctic Ocean Gas Hydrate Stability in a Changing Climate. J Geol Res 2013:1-10. https://doi.org/10.1155/2013/783969

Goecks J, Nekrutenko A, Taylor J, et al (2010) Galaxy: a comprehensive approach for supporting accessible, reproducible, and transparent computational research in the life sciences. Genome Biol 11:. https://doi.org/10.1186/gb-2010-11-8-r86

Ho A, De Roy K, Thas O, et al (2014) The more, the merrier: Heterotroph richness stimulates methanotrophic activity. ISME J 8:1945-1948. https://doi.org/10.1038/ismej.2014.74

Huerta-Cepas J, Szklarczyk D, Forslund K, et al (2016) eggNOG 4.5: A hierarchical orthology framework with improved functional annotations for eukaryotic, prokaryotic and viral sequences. Nucleic Acids Res 44:D286-D293. https://doi.org/10.1093/nar/gkv1248

Hunter SJ, Goldobin DS, Haywood AM, et al (2013) Sensitivity of the global submarine hydrate inventory to scenarios of future climate change. Earth Planet Sci Lett 367:105-115.

https://doi.org/10.1016/j.epsl.2013.02.017 
Iguchi H, Yurimoto H, Sakai Y (2011) Stimulation of methanotrophic growth in cocultures by cobalamin excreted by rhizobia. Appl Environ Microbiol 77:8509-8515. https://doi.org/10.1128/AEM.05834-11

Jadav S, Sakthipriya N, Doble M, Sangwai JS (2017) Effect of biosurfactants produced by Bacillus subtilis and Pseudomonas aeruginosa on the formation kinetics of methane hydrates. J Nat Gas Sci Eng 43:156-166. https://doi.org/10.1016/j.jngse.2017.03.032

Jeon YS, Lee K, Park SC, et al (2014) EzEditor: A versatile sequence alignment editor for both rRNA- and protein-coding genes. Int J Syst Evol Microbiol 64:689-691. https://doi.org/10.1099/ijs.0.059360-0

Jhala YK, Vyas R V., Shelat HN, et al (2014) Isolation and characterization of methane utilizing bacteria from wetland paddy ecosystem. World J Microbiol Biotechnol 30:1845-1860.

https://doi.org/10.1007/s11274-014-1606-3

Kanehisa M, Sato Y, Kawashima M, et al (2016) KEGG as a reference resource for gene and protein annotation. Nucleic Acids Res 44:D457-D462. https://doi.org/10.1093/nar/gkv1070

Ketzer M, Praeg D, Pivel MAG, et al (2019) Gas seeps at the edge of the gas hydrate stability zone on Brazil's continental margin. Geosci 9:1-11. https://doi.org/10.3390/geosciences 9050193

Ketzer M, Praeg D, Rodrigues LF, et al (2020) Gas hydrate dissociation linked to contemporary ocean warming in the southern hemisphere. Nat Commun 11:. https://doi.org/10.1038/s41467-020-17289-z

Kumar S, Stecher G, Li M, et al (2018) MEGA X: Molecular evolutionary genetics analysis across computing platforms. Mol Biol Evol 35:1547-1549. https://doi.org/10.1093/molbev/msy096

Lee J, Shin NR, Jung MJ, et al (2013) Paenibacillus oceanisediminis sp. nov. isolated from marine sediment. Int J Syst Evol Microbiol 63:428-434. https://doi.org/10.1099/ijs.0.037085-0

Marchler-Bauer A, Bryant SH (2004) CD-Search: Protein domain annotations on the fly. Nucleic Acids Res 32:W327-W331. https://doi.org/10.1093/nar/gkh454

Medina-Silva R, de Oliveira RR, Pivel MAG, et al (2018a) Microbial diversity from chlorophyll maximum, oxygen minimum and bottom zones in the southwestern Atlantic Ocean. J Mar Syst 178:52-61. https://doi.org/10.1016/j.jmarsys.2017.10.008

Medina-Silva R, Oliveira RR, Trindade FJ, et al (2018b) Microbiota associated with tubes of Escarpia sp. from cold seeps in the southwestern Atlantic Ocean constitutes a community distinct from that of surrounding marine sediment and water. Antonie Van Leeuwenhoek 111:533-550. https://doi.org/doi.org/10.1007/s10482-017-0975-7

Miller DJ, Ketzer JM, Viana AR, et al (2015) Natural gas hydrates in the Rio Grande Cone (Brazil): A new province in the western South Atlantic. Mar Pet Geol 67:187-196.

https://doi.org/10.1016/j.marpetgeo.2015.05.012

Page 15/23 
Niemann H, Linke P, Knittel K, et al (2013) Methane-Carbon Flow into the Benthic Food Web at Cold Seeps - A Case Study from the Costa Rica Subduction Zone. PLoS One 8:4-13.

https://doi.org/10.1371/journal.pone.0074894

Okonechnikov K, Golosova O, Fursov M, et al (2012) Unipro UGENE: A unified bioinformatics toolkit. Bioinformatics 28:1166-1167. https://doi.org/10.1093/bioinformatics/bts091

Piñero E, Marquardt M, Hensen C, et al (2013) Estimation of the global inventory of methane hydrates in marine sediments using transfer functions. Biogeosciences 10:959-975. https://doi.org/10.5194/bg-10959-2013

Pitcher DG, Saunders NA, Owen RJ (1989) Rapid extraction of bacterial genomic DNA with guanidium thiocyanate. Lett Appl Microbiol 8:151-156. https://doi.org/10.1111/j.1472-765X.1989.tb00262.x

R Core Team (2017) R: A language and environment for statistical computing

Rodrigues LF, Ketzer JM, Lourega RV, et al (2017) The influence of methane fluxes on the sulfate/methane interface in sediments from the Rio Grande Cone Gas Hydrate Province, southern Brazil. Brazilian J Geol 47:369-381. https://doi.org/10.1590/2317-4889201720170027

Rogers RE, Kothapalli C, Lee MS, Woolsey JR (2003) Catalysis of gas hydrates by biosurfactants in seawater-saturated sand/clay. Can J Chem Eng 81:973-980. https://doi.org/10.1002/cjce.5450810508

Ross MO, Rosenzweig AC (2017) A tale of two methane monooxygenases. J Biol Inorg Chem 22:307319. https://doi.org/10.1007/s00775-016-1419-y

Singh R, Ryu J, Kim SW (2019) Microbial consortia including methanotrophs: some benefits of living together. J Microbiol 57:939-952. https://doi.org/10.1007/s12275-019-9328-8

Stock M, Hoefman S, Kerckhof FM, et al (2013) Exploration and prediction of interactions between methanotrophs and heterotrophs. Res Microbiol 164:1045-1054.

https://doi.org/10.1016/j.resmic.2013.08.006

Takishita K, Kakizoe N, Yoshida T, Maruyama T (2010) Molecular evidence that phylogenetically diverged ciliates are active in microbial mats of deep-sea cold-seep sediment. J Eukaryot Microbiol 57:76-86. https://doi.org/10.1111/j.1550-7408.2009.00457.x

Tatusova T, DiCuccio M, Badretdin A, et al (2016) NCBI prokaryotic genome annotation pipeline. Nucleic Acids Res 44:6614-6624. https://doi.org/10.1093/nar/gkw569

Vallotton P (2013) Size matters: Filamentous bacteria drive interstitial vortex formation and colony expansion in Paenibacillus vortex. Cytom Part A 83A:1105-1112. https://doi.org/10.1002/cyto.a.22354 
Veraart AJ, Garbeva P, Van Beersum F, et al (2018) Living apart together - Bacterial volatiles influence methanotrophic growth and activity. ISME J 12:1163-1166. https://doi.org/10.1038/s41396-018-0055-7

Wasi S, Tabrez S, Ahmad M (2013) Use of Pseudomonas spp. for the bioremediation of environmental pollutants: A review. Environ Monit Assess 185:8147-8155. https://doi.org/10.1007/s10661-013-3163-x

Wörmer L, Hoshino T, Bowles MW, et al (2019) Microbial dormancy in the marine subsurface: Global endospore abundance and response to burial. Sci Adv 5:24-26. https://doi.org/10.1126/sciadv.aav1024

Yoon SH, Ha SM, Kwon S, et al (2017) Introducing EzBioCloud: A taxonomically united database of 16S rRNA gene sequences and whole-genome assemblies. Int J Syst Evol Microbiol 67:1613-1617. https://doi.org/10.1099/ijsem.0.001755

Zheng L, Yi Y, Liu J, et al (2014) Isolation and characterization of marine Brevibacillus sp. S-1 collected from South China sea and a novel antitumor peptide produced by the strain. PLoS One 9:7-14. https://doi.org/10.1371/journal.pone.0111270

Zheng R, Wu S, Ma N, Sun C (2018) Genetic and Physiological Adaptations of Marine Bacterium Pseudomonas stutzeri 273 to Mercury Stress. Front Microbiol 9:.

https://doi.org/10.3389/fmicb.2018.00682

\section{Figures}




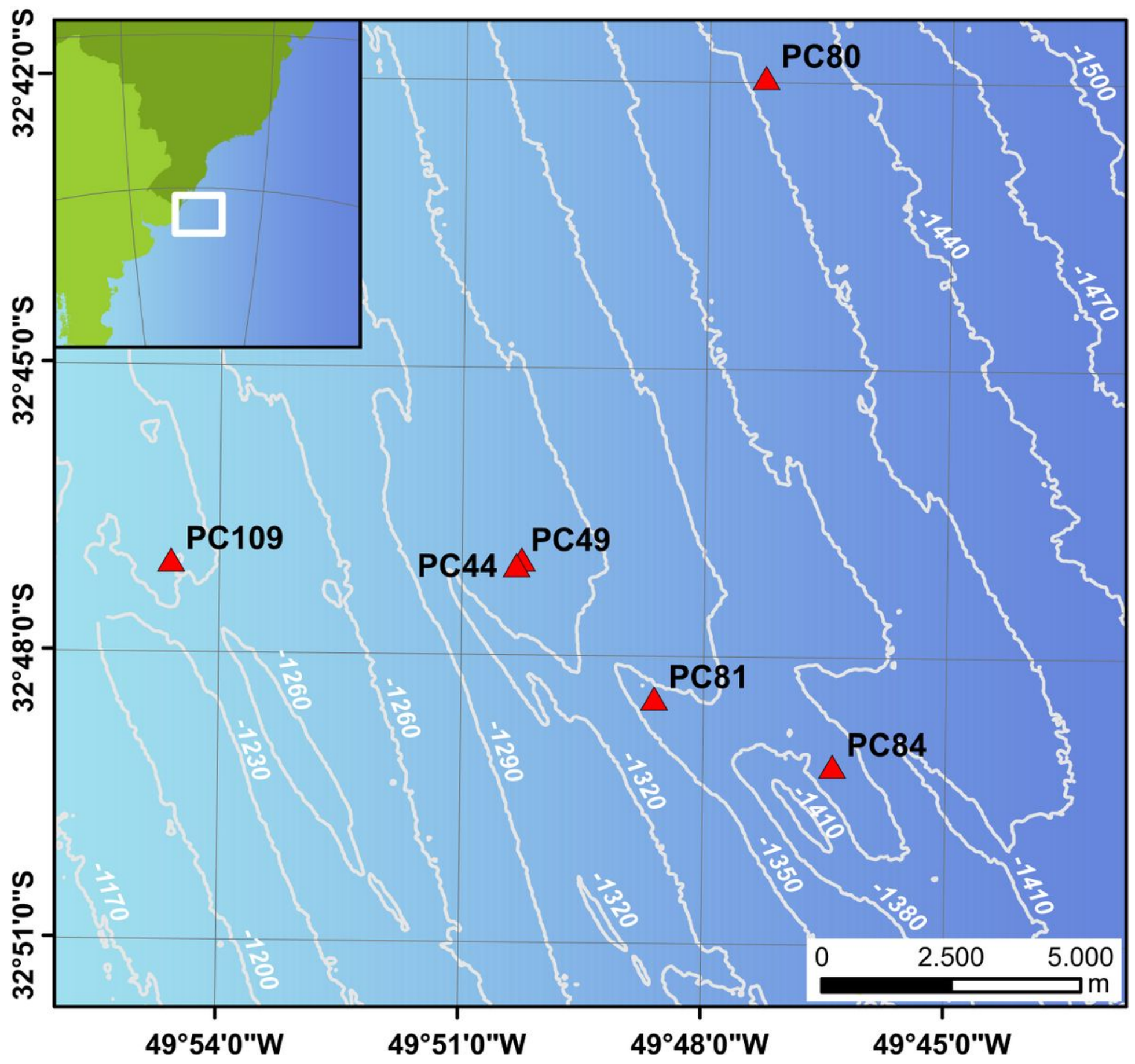

Figure 1

Map of sediment sampling sites at the Rio Grande Cone, Pelotas Basin, located in the western South Atlantic. Bathymetric information were obtained from Miller et al. (17). Sample collections were performed during two oceanographic expeditions, MR11 (2011; PC44, DR44, and PC49) and MD195 (2013; PC80, PC81, PC84 and PC109) 


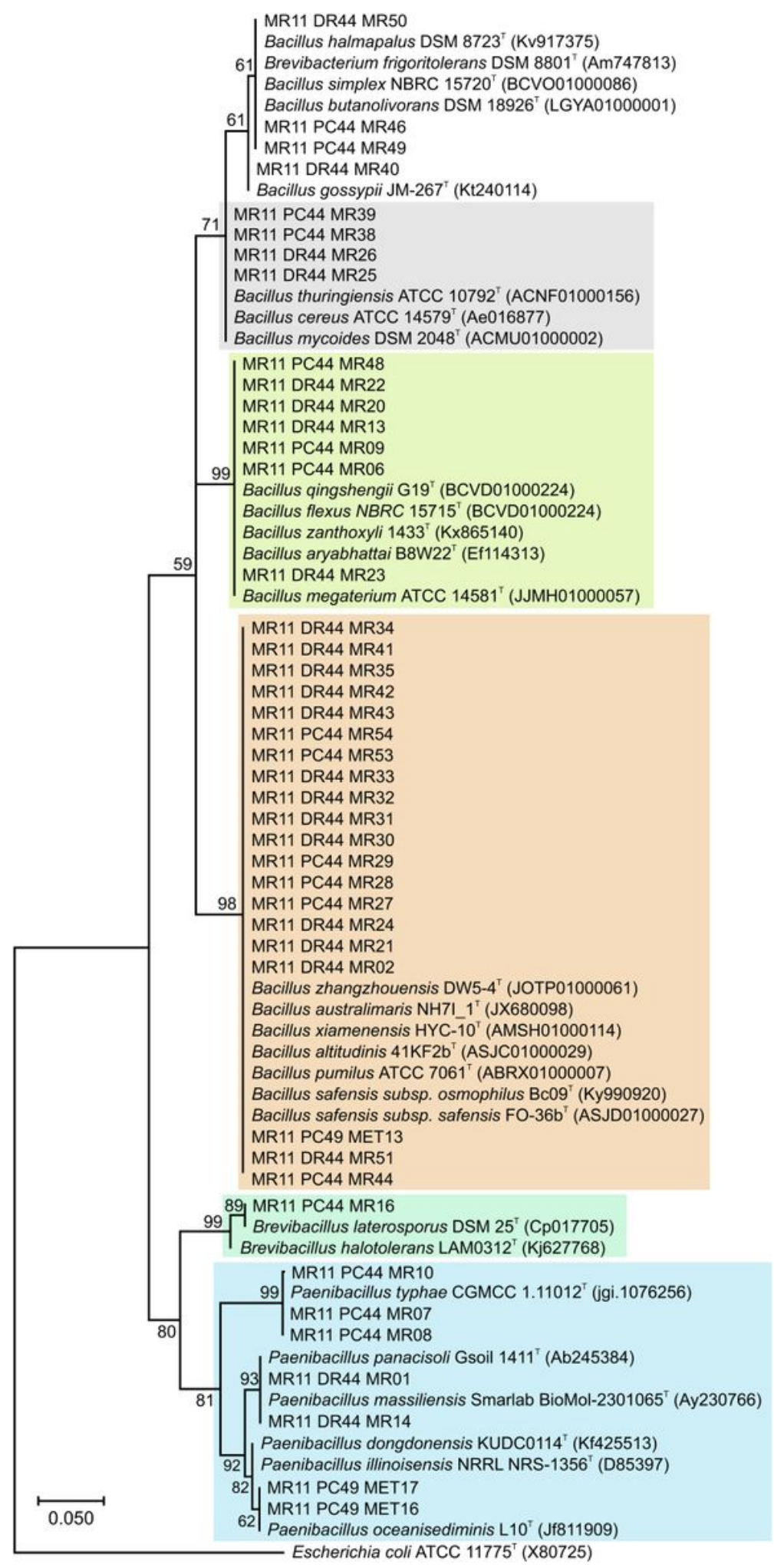

Figure 2

Phylogenetic tree based on partial 16S rRNA sequences from PC44, DR44 and PC49 isolates. PC44 and DR44 represent collections from a site with associated benthic fauna, while PC49 showed the presence of gas hydrates. Phylogeny constructed by the Maximum Likelihood method and Kimura 2-parameter model, with node values based on 1 000-replication bootstrap. Escherichia coli was used as outgroup 


MD195 PC80 121
MD195 PC80 122
MD195 PC80 123
MD195 PC80 124
MD195 PC81 125
MD195 PC81 126
MD195 PC81 127
MD195 PC81 128
MD195 PC81 129
MD195 PC81 130
MD195 PC81 131
MD195 PC81 132
MD195 PC84 133
MD195 PC109 134

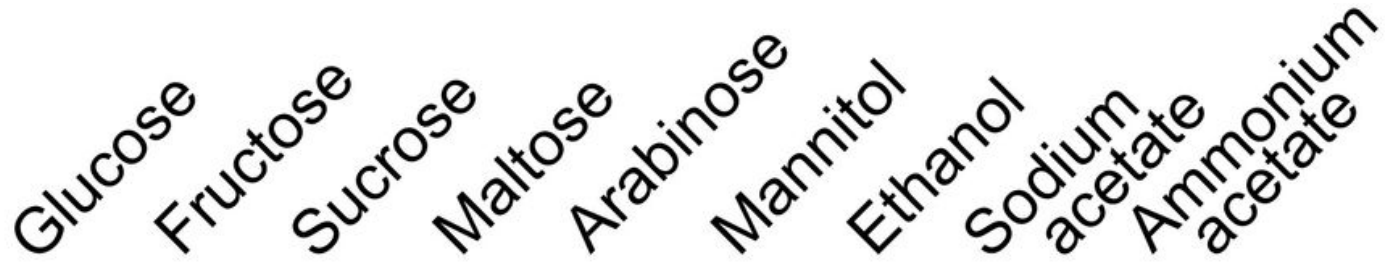

Figure 3

Ability of isolates obtained under methane atmosphere to metabolize multicarbon compounds (saccharides: glucose, fructose, sucrose, maltose, arabinose; sugar-alcohol: mannitol; alcohol: ethanol; acetate: ammonium acetate) after 48 -hour incubation at $25^{\circ} \mathrm{C}$. Open circles indicate no growth. Eight isolates (highlighted) produced fluorescent pigments during assay. Data were based on three replicates 


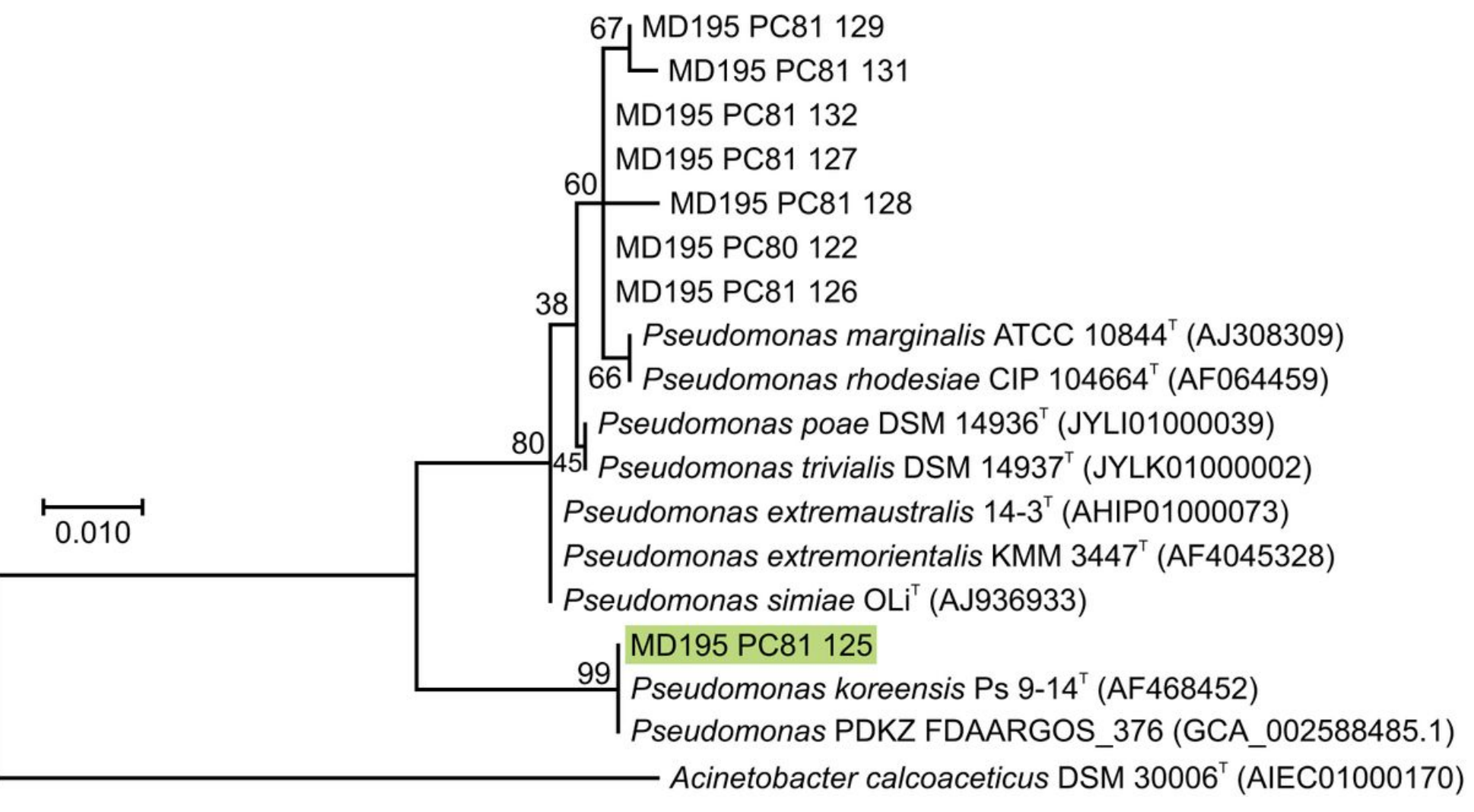

Figure 4

Maximum Likelihood phylogenetic tree of eight Pseudomonas sp. isolates, comparing 16S rRNA sequences with closest relatives. The tree was inferred using Kimura 2-parameter model and node values evaluated through a 1000x bootstrap. Acinetobacter calcoaceticus was used as outgroup. The isolate MD195-PC81-125 (highlighted) was further investigated through whole genome sequencing 


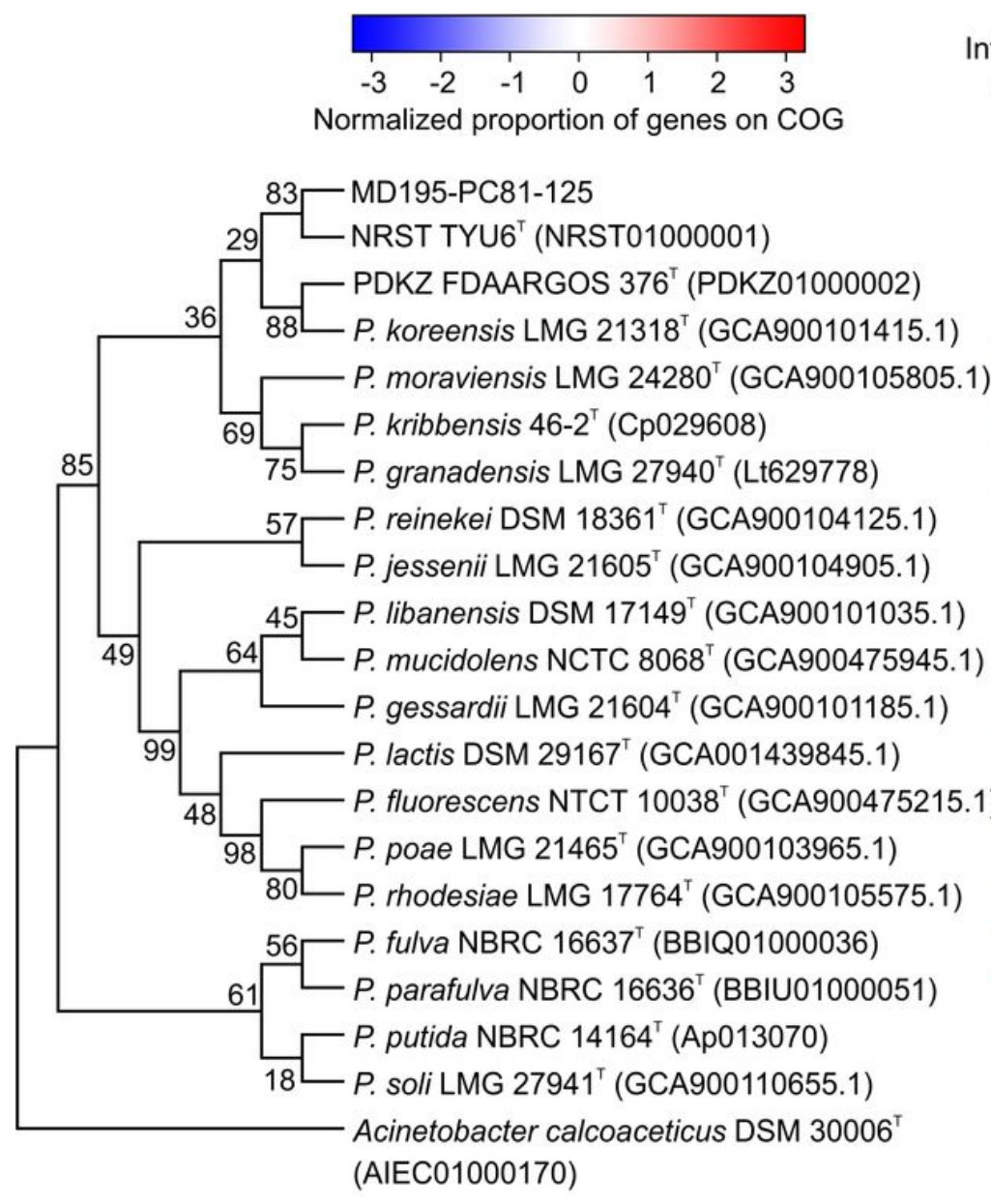

Information storage

and processing Metabolism

Cellular processes and signaling

\section{Information storage and processing}

A RNA processing and modification

B Chromatin structure and dynamics

J Translation and ribosomal structure

K Transcription

L Replication and repair

S Function unknown
Metabolism

C Energy production and conversion

E Amino acid transp. and met.

F Nucleotide transp. and met.

G Carbohydrate transp. and met.

H Coenzyme transp. and met.

I Lipid transp. and met.

P Inorganic ion transp. and met.

Q Secondary metabolites biosynthesis, transport and catabolism
Cellular processes and signaling

D Cell cylce control and cell division

M Cell wall/membrane/envelope biogenesis

N Cell motility

O Post-translational modification, protein turnover, chaperone functions

T Signal transduction mechanisms

U Intracellular trafficking and secretion

V Defense mechanisms

Z Cytoskeleton

\section{Figure 5}

Genome analysis of Pseudomonas MD195-PC81-125. Pseudomonas spp. type strains ordered according to 16S rRNA gene phylogenetic analysis (left) and compared through the proportion of encoded genes belonging to each COG class (right). Heatmap colors were scaled per COG column. (*) Indicates COG (Clusters of Orthologous Genes) classes in which MD195-PC81-125 differed significantly from gene frequency distributions of the other strains

\section{Supplementary Files}

This is a list of supplementary files associated with this preprint. Click to download. 
- ProencaetalSupplementarymaterials.pdf 